\title{
Non-local rings whose ideals are all quasi-injective
}

\section{G. Ivanov}

\begin{abstract}
A ring is a left $Q$-ring if all of its left ideals are quasi-injective. For an integer $m \geq 2$, a sfield $D$, and a null $D$-algebra $V$ whose left and right $D$-dimensions are both equal to one, let $H(m, D, V)$ be the ring of all $m \times m$ matrices whose only non-zero entries are arbitrary elements of $D$ along the diagonal and arbitrary elements of $V$ at the places $(2,1), \ldots,(m, m-1)$ and $(1, m)$. We show that the only indecomposable non-local left Q-rings are the simple artinian rings and the rings $H(m, D, V)$. An arbitrary left Q-ring is the direct sum of a finite number of indecomposable non-local left $Q$-rings and a $Q$-ring whose idempotents are all central.
\end{abstract}

A ring is a (Zeft) Q-ring if all of its left ideals are quasi-injective. The study of Q-rings was initiated in [2]; in this note we determine the structure of indecomposable non-local Q-rings, and reduce the general problem to the investigation of Q-rings whose idempotents are all central. We show that there are only two types (both artinian) of indecomposable non-local Q-rings, and represent them as matrix rings. our methods depend on the existence of non-central idempotents, a condition satisfied by all indecomposable non-local Q-rings, since the local Q-rings are precisely those with no idempotents other than their identities [1, Proposition 5.8].

Throughout this note all rings are unital and associative; all

Received 16 August 1971. Communicated by Hanna Neumann. The author would like to thank his supervisor, Professor Hanna Neumann, for her help in the preparation of this paper. 
Q-rings are indecomposable and non-local; all modules are unital; and everything is done on the left (unless otherwise specified), so, for example, ideal means left ideal. The socle of a module $M$ is denoted by $S(M)$.

We begin by showing that a Q-ring has non-zero socle: this guarantees that the ring has primitive idempotents. We then show that the ring cannot have an infinite number of ideals with the following two properties: the sum of the ideals is direct, and each ideal has a homomorphic image in the ring which intersects the ideal trivially. Using this we deduce that if at least one minimal ideal is injective then the ring is simple artinian. If the ring is not simple artinian then every indecomposable injective ideal has a unique proper submodule. It follows that the socle of the ring is essential. We then show that the ring is artinian and represent it as a full ring of matrices.

We will need the following result.

LEMMA 1 [2, Theorem 2.3]. A ring is a Q-ring if, and only if, it is self-injective and all of its essential ideals are two-sided.

LEMMA 2. The socie of a Q-ring $R$ is non-zero. If $e_{1}, e_{2} \in R$ are orthogonal idempotents, then $e_{2} R e_{1} \subseteq S\left(R e_{1}\right)$. If $R e_{1} \cong R e_{2}$ then both $R e_{i}$ are (finite) sums of minimal ideals.

Proof. Let $e, f \in R$ be orthogonal idempotents whose sum is the identity. As $R$ is indecomposable, either $e R f \neq 0$ or $f R e \neq 0$. Assume the latter and let $L$ be an essential submodule of $R e$. By Lemma 1 , the ideal $L \oplus R f$ is two-sided: consequently $f R e=f^{2} R e$ is contained in $L$. Therefore the intersection $L_{0}$ of all essential submodules of $R e$ is non-zero: it follows that $L_{0}$ is a sum of minimal ideals and so the socle of $R$ is non-zero. By a similar argument we can show that $e_{2} R e_{1} \subseteq S\left(R e_{1}\right)$. If $R e_{1} \cong R e_{2}$ then $S\left(R e_{1}\right)$ contains $e_{2} R e_{1}$ which generates $R e_{1}$ : therefore $R e_{1}=S\left(R e_{1}\right)$.

LEMMA 3. Let $R$ be $a$ Q-ring and let $\left\{e_{i} \mid i \in I\right\}$ be an infinite set of mutually orthogonal idempotents. Then only finitely many $e_{i}$ have the property that $R e_{i}$ has a homomorphic image in $R\left(1-e_{i}\right)$. 
Proof. Let $I_{0}$ be the set of all $i \in I$ such that $R e_{i}$ has a homomorphic image in $R\left(1-e_{i}\right)$, and assume that $I_{0}$ is infinite. If any two ideals $R e_{i}, R e_{j}, i, j \in I$, have a common homomorphic image then, by the projectivity of $R e_{i}$, there is a homomorphism from $R e_{i}$ to $R e_{j}$. It follows that there is an infinite subset $I_{1} \subseteq I_{0}$ with the property that for each $i \in I_{1}$ there is a homomorphism $\varphi_{i}: R e_{i} \rightarrow R\left(1-e_{i}\right)$ satisfying the conditions that $\left({ }_{I_{1}}^{\oplus} R_{i}\right) \cap R e_{i} e_{i}=0$ and that all the $R e_{i}{ }_{i}$ are distinct. Let $R e$ be an injective hull of $\oplus_{I_{l}} R e_{i}$ and let $\varphi: \underset{I_{1}}{\oplus} R e_{i}+R(1-e)$ be the sum of all the $\varphi_{i}, i \in I_{1}$. As $R(1-e)$ is injective, $\varphi$ can be lifted to a homomorphism $\bar{\varphi}: R e \rightarrow R(1-e)$. But this is a contradiction since, by Lemma $2, \operatorname{Re} \bar{\varphi}$ is a finite sum of minimal ideals. Hence $I_{0}$ is finite.

THEOREM 1. Let $R$ be a Q-ring and $e \in R$ an idempotent. If Re is a minimal ideal then $R$ is a simple artinian ring.

Proof. By Lemma 3 the ring $R$ has only a finite number of ideals isomorphic to $R e$. Let $R f$ be their sum, then $f R(1-f)=0$. Since $R e$ is projective it can be a homomorphic image only of modules which contain an isomorphic copy of itself. Therefore $(1-f) R f=0$. As $R$ is indecomposable this means that it is a sum of mutually isomorphic minimal ideals. Therefore $R$ is a simple artinian ring.

In view of Theorem 1 all Q-rings will, from now on, have the property that none of their minimal ideals is injective.

LEMMA 4. Let $e$ be a primitive idempotent in a Q-ring $R$. Then eRe is a sfield and the only submodule of $R e$ is $S(R e)=(1-e) R e$.

Proof. First we will show that $(1-e) R e \neq 0$. Assume that $(1-e) R e=0$; then the ideals of $e R e$ and the $R$-submodules of $R e$ coincide, and as $R$ is indecomposable, $e R(1-e) \neq 0$. By Proposition 5.8 of [1] the ring $e R e$ has a unique maximal ideal, $J$ say, which is not zero since $R$ has no minimal ideals which are injective. Therefore there is an ideal $L \subseteq J$ which has a simple factor module. As $e R e$ is a local 
ring and as every image in $R(1-e)$ of $R e$ is a minimal ideal (Lemma 2), this means that there is a homomorphism from $L$ to $R(1-e)$. By the injectivity of $R(1-e)$ this map can be lifted to $R e$ : a contradiction, since by Lemma 2 every homomorphism from $R e$ to $R(1-e)$ kills $J$ and hence $L$. Therefore $(1-e) R e \neq 0$.

By Theorem 5.1 of [1] every element of $J$ is annihilated on the left by an essential submodule of $R e$. Hence, by Lemma 2, (1-e)ReJ $=0$ and so $J$ is an ideal of $R$. But every non-zero submodule of $R e$ must contain the minimal ideal $S(R e) \supseteq(1-e) R e$; therefore $J=0$. That is, $e R e$ is a sfield. Hence every non-zero element of eRe generates Re and, as $(1-e) R e$ generates $S(R e)$, the only submodule of $R e$ is $S(R e)=(1-e) R e$.

LEMMA 5. The socle of a Q-ring is essential.

Proof. Let $R$ be a Q-ring and let $R e$ be an injective hull of $S(R)$. If $f_{1}, f_{2} \in R(1-e)$ are orthogonal idempotents then, by Lemma 2 , both $f_{1} R f_{2}=0$ and $f_{2} R f_{1}=0$ : so as $R$ is indecomposable, both products $f_{i} R e \neq 0, i=1,2$. As $R(1-e)$ does not contain any minimal ideals it does not contain any primitive idempotents (Lemma 4 ). Therefore $R(1-e)$ has an infinite set $\left\{e_{i}\right\}$ of mutually orthogonal idempotents such that each $R e_{i}$ has a homomorphic image in $R e:$ a contradiction to Lemma 3. Therefore $R(1-e)=0$ and $S(R)$ is essential in $R$.

An idempotent is finite if it is a (finite) sum of primitive orthogonal idempotents; otherwise it is infinite.

THEOREM 2. A Q-ring is artinian.

Proof. Let $R$ be a $Q$-ring and assume it is not artinian. Then, by Lemmas 4 and 5 , the set $\left\{M_{i} \mid i \in I\right\}$ of minimal ideals of $R$ is infinite. For each $i \in I$ let $R e_{i}$ be an injective hull of $M_{i}$. It follows from Lemmas 2,3 and 4 that only a finite number of the $R e_{i}$, say $R e_{t(1)}, \ldots, R e_{t(n)}$, have proper homomorphic images in $R$, and that these are minimal ideals, say $M_{s(I)}, \ldots, M_{s(n)}$. Let $I_{0}=I \backslash\{t(1), \ldots, t(n)\} ;$ then $e_{i}^{M_{j}}=0$ for every pair 
$(i, m) \in \ddot{\ddot{O}_{0}} \times I$

As all the $M_{i}$ are mutually non-isomorphic minimal ideals it follows that for every pair $(i, j) \in I \times I$ there is an element $x \in 2\left(M_{j}\right)$, the left annihilator of $M_{j}$, with the property that $x M_{i} \neq 0$. By Theorem 5.1 of [1], there is an element $y \in R$ such that $y x$ is an idempotent modulo the Jacobson radical $J$ of $R$ with the property that $x+J=r y x+J$ for some $r \in R$. By Theorem 5.6 of [1] there is an idempotent $f \in R$ such that $f+J=y x+J$. Therefore $f \in Z\left(M_{j}\right)$ and $f M_{i}=M_{i}$, that is, $M_{i}$ is an image of $R f$. This enables us to pick out an infinite number of orthogonal idempotents with the property that the ideal generated by each idempotent has an image outside itself, thus contradicting Lemma 3 .

Let $e^{\prime}, e^{\prime \prime}$ be infinite orthogonal idempotents whose sum is the identity and having the property that all the ideals $R e_{t(1)}, \ldots, \operatorname{Re}_{t(n)}$, $M_{s(1)}, \ldots, M_{s(n)}$ are contained in one and the same of the ideals $R e^{\prime}, R e^{\prime \prime}$. Then there are an infinite number of minimal ideals which are images of one of the ideals $R e^{\prime}, R e^{\prime \prime}$ and are contained in the other. For assume that this is not true, and let $R e_{1}^{\prime}$ (respectively $R e_{1}^{\prime \prime}$ ) be an injective hull in $R e^{\prime \prime}$ (respectively $R e^{\prime}$ ) of all the images of $R e^{\prime}$ (respectively $R e^{\prime \prime}$ ) in $R e^{\prime \prime}$ (respectively $R e^{\prime}$ ). As the idempotents $e_{1}^{l}, e_{1}^{\prime \prime}$ are both finite the ideals $R e_{1}^{1}, R e_{1}^{\prime \prime}$ have no images outside themselves in $R:$ therefore the ideals $R\left(e^{\prime}+e_{1}^{\prime}-e_{1}^{\prime \prime}\right)$ and $R\left(e^{\prime \prime}+e_{1}^{\prime \prime}-e_{1}^{\prime}\right)$ annihilate each other. As $R$ is indecomposable and $R=R\left(e^{\prime}+e_{1}^{\prime}-e_{1}^{\prime \prime}\right) \oplus R\left(e^{\prime \prime}+e_{1}^{\prime \prime}-e_{1}^{\prime}\right)$, this is impossible: therefore one of the ideals, $R e^{\prime}$ say, has an infinite number of images in the other, $R e^{\prime \prime}$. Let $I_{1}=\left\{i \in I_{0} \mid e^{\prime} M_{i}=M_{i}\right.$ and $\left.M_{i} \subseteq R e^{\prime \prime}\right\}$. Let $k \in I_{1}$, and let $g \in Z\left(M_{k}\right) \cap R e^{\prime}$ be an idempotent which is a left identity for some $M_{i}$, $i \in I_{1}$. Then one of the idempotents $g, e^{\prime}-g$ is a left identity for an infinite number of $M_{i}, i \in I_{1}$. Denote $i t$ by $f_{1}^{\prime}$ and let the other idempotent be $f_{1}$. By a completely analagous argument we can show that $f_{1}^{\prime}$ is a sum of two orthogonal idempotents $f_{2}, f_{2}^{\prime}$ such that $f_{2}^{\prime}$ is a 
left identity for an infinite number of $M_{i}, i \in I_{1}$. As this procedure is clearly inductive, we can pick for each positive integer $n$ an idempotent $f_{n} \in R e^{\prime}$ such that all the $f_{n}$ are mutually orthogonal and each $R f_{n}$ has an image in $R e^{\prime \prime}$. But this is a contradiction to Lemma 3: therefore our original assumption is false. That is, $R$ is artinian.

For any integer $m \geq 2$, any sfield $D$, and any null $D$-aigebra $V$ whose left and right $D$-dimensions are both equal to one, let $H(m, D, V)$ be the ring of all $m \times m$ matrices whose only non-zero entries are arbitrary elements of $D$ along the diagonal, and arbitrary elements of $V$ at the places $(2,1), \ldots,(m, m-1)$ and $(1, m)$.

THEOREM 3. Every Q-ring is isomorphic to one of the rings $H(m, D, V)$; conversely, every $H(m, D, V)$ is a Q-ring.

Proof. Let $R$ be a Q-ring; then, by Theorem 2 and Lemma 4, there is an integer $m \geq 2$ and a set $\left\{e_{i} \mid 1 \leq i \leq m\right\}$ of mutually orthogonal primitive idempotents such that $R=\bigoplus_{1}^{m} R e_{i}$. As the only minimal ideals of $R$ are the socles of the $R e_{i}$, it follows from Lemma 4 that every minimal ideal is the image of an $R e_{i}$. If a minimal ideal $M$ is the image of an $R e_{i}$, then as $R e_{i}$ is projective it follows from Lemme 2 that $M$ is not the image of any other $R e_{j}$, that is, $e_{j} M=0$ for all $j \neq i$. Therefore each $R e_{i}$ determines uniquely that $R e_{j}$ whose socle is the image of $R e_{i}$ and that $R e_{k}$ which has $S\left(R e_{i}\right)$ as a factor module. As $R$ is indecomposable a standard argument shows that we may assume the $e_{i}$ to be indexed in such a way that $s\left(R e_{i}\right)$ is the image of $R e_{i+1}$ if $1 \leq i \leq m-1$ and $S\left(R e_{m}\right)$ is the image of $R e_{1}$.

We know that $R \cong \operatorname{hom}_{R}(R, R)$ which is isomorphic to $H$, say, the ring of all $m \times m$ matrices $\left(\varphi_{i j}\right)$ where $\varphi_{i j} \in \operatorname{hom}_{R}\left(R e_{i}, R e_{j}\right)=H_{i j}$. It follows from the preceding paragraph that the only non-zero entries of $H$ are along the diagonal and at the places $(2,1), \ldots,(m, m-1)$ and $(1, m)$. As each $R e_{i}$ is injective and $e_{i} R e_{i}$ is a sfield, it follows 
that $H_{i i} \cong \operatorname{nom}_{R}\left(S\left(R e_{i}\right), S\left(R e_{i}\right)\right)$. If $S\left(R e_{i}\right)$ is the image of $R e_{j}$ then ${ }_{j j} \cong \operatorname{hom}_{R}\left(S\left(R e_{i}\right), S\left(R e_{i}\right)\right)$, therefore all the sfields $H_{i i}$ are mutually isomorphic; $H_{i i} \cong D$, say, for all $i$. Moreover, each non-zero $H_{i j}$ is a one dimensional left vector space over $H_{i i}$ and a one dimensional right vector space over $H_{i j}$. Hence all the non-zero $H_{i j}$ are mutually isomorphic $D$-bivector spaces, all isomorphic to $V$, say. As $H_{i j}{ }_{j k}=0$, the space $V$ is a null algebra. Therefore $R \cong H(m, D, V)$.

We now prove the converse: we show that $H=H(m, D, V)$ is a Q-ring. First we show that $H$ is injective. For any $i$ let $e_{i} \in H$ be the matrix whose only non-zero entry is 1 at the place $(i, i)$. If $L \subseteq H$ is an ideal then $L=L_{1} \oplus L_{2}$ where $L_{1} \subseteq H e_{i}$ and $L_{2} \cap H e_{i}=0$. Any homomorphism from $L_{1}$ to $H_{i}$ can be lifted to an endomorphism of $\mathrm{He}_{i}$. If there is a homomorphism $\varphi$ from $L_{2}$ to $\mathrm{He}_{i}$ then $L_{2}$ contains a direct summand isomorphic to $H e_{j}(j=i+1$ if $i \neq m ; j=1$ if $i=m)$, and ker $\varphi$ is a complement to this summand. Consequently every homomorphism from $L$ to $H e_{i}$ can be lifted to $H$, that is, $H e_{i}$ is injective. Therefore, as $H$ is a finite sum of injective ideals, it is itself injective. For any element $x \in H$ it is clear that $x H \subseteq S(H)+H x$. Therefore every essential ideal of $H$ is two-sided. Now we apply Lemma 1 to deduce that $H$ is a Q-ring.

REMARK. It follows from Lerma 3 that an arbitrary Q-ring (without the restrictions imposed in this note) is the sum of a finite number of artinian non-local Q-rings (as described in Theorems 1 and 3 ) and a Q-ring whose idempotents are all central.

\section{References}

[1] Carl Faith, Lectures on injective modules and quotient rings (Lecture Notes in Mathematics, 49. Springer-Verlag, Berlin, Heidelberg, New York, 1967). 
[2] S.K. Jain, S.H. Mohamed and Surjeet Singh, "Rings in which every right ideal is quasi-injective", Pacific J. Math. 31 (1969), 73-79.

Department of Pure Mathematics, School of General Studies, Australian National University, Canberra, ACT. 